\title{
Efficacy of transdermal diclofenac patches in the treatment of pain associated with various temporomandibular joint disorders: A comparative study
}

\author{
Altaf Hussain ${ }^{1}$, Rayees Ahmad², Nusrat Nazir ${ }^{3 *}$ \\ ${ }^{1}$ Professor and HOD, ${ }^{2}$ PG Student, ${ }^{3}$ Senior Resident, Dept. of Oral Medicine \& Radiology, Government Dental College, Srinagar, Jammu \\ Kashmir, India
}

*Corresponding Author: Nusrat Nazir

Email: drnusratnaziromar@gmail.com

\begin{abstract}
Introduction: Temporomandibular disorders (TMD) is a term used to describe collectively a number of related disorders affecting the temporomandibular joints, masticatory muscles, and associated structures. The treatment modalities include surgical and non-surgical methods. In this study non-surgical method was used. The aim of present study was to evaluate and compare the efficacy of topical and systemic NSAIDs in the management of pain of TMD origin.

Materials and Methods: A single blinded cross-sectional study was conducted and a total of 40 clinically diagnosed cases of TMDs of muscular origin were selected. Once the diagnosis of myalgia due to TMD was made, the patients were randomly allocated in one of the treatment groups. The study divided the participants into 2 categories: GROUP A: oral diclofenac 50mg, and Thiocholchicocide $4 \mathrm{mg} 12$ hourly for 15 days. GROUP B: 200mg of transdermal patch of diclofenac to be applied over the masseter muscle 12 hourly and Thiocolchicoside $4 \mathrm{mg}$ twice daily as in group A for the period of 15 days.

Results: Females where most commonly affected with a ratio of 2.07:1 and a mean age of 33.5 years. For group A mean verbal rating score of pain was 8, 5, 4, 1 on 3rd, 7th, 15th, and 21ist day respectively and group B mean verbal rating score of pain was 7, 5, 3, 1 on 3rd, 7 th, 15th, and 21ist day respectively. There was no significant difference in trends of pain intensity in patients treated by oral NSAIDS and patients who were treated with Transdermal patch group.

Conclusion: There was no significant difference in trends of pain intensity in patients treated by oral NSAIDS and patients who were treated with Transdermal patch group. However treatment with topical NSAIDs has been shown to provide clinically effective analgesia at the site of application while minimizing systemic absorption.
\end{abstract}

Keywords: Transdermal diclofenac patch, Thiocholchicoside, Gamma amino butyric acid, Temporomandibular joint disorders.

\section{Introduction}

Temporomandibular disorders (TMD) is a term that includes a group of related disorders affecting the temporomandibular joints, muscles of mastication, and associated structures, they present with common symptoms like pain, tenderness and limited mouth opening. ${ }^{1}$ The most widely accepted definition for TMD is "it is a group of musculoskeletal conditions that involve the temporomandibular joints (TMJs), the masticatory muscles and all associated tissues". ${ }^{2}$ MPDS is said to be present when a patient has all the four cardinal symptoms, i.e., pain, tenderness, clicking and limited mouth opening. These symptoms are mainly due to fatigue and spasm of masticatory muscles. ${ }^{3}$ The characteristic presentation of MPDS is regional pain originating from hyperirritable foci located within painful taut bands of skeletal muscle, known as myofacial trigger points. The main causes of MPDS include direct or indirect trauma, spine pathology, exposure to cumulative and repetitive strain, postural dysfunction, and physical deconditioning. The presentation of TMDs includes, temporomandibular joint pain or masticatory muscle pain during chewing, which may radiate or refer to local or distant associated structures of the joint, sounds like clicking (on opening or closing or both), popping, or crepitus (a continuous grating sound) of the temporomandibular joint on any of its movements with or without locking of the joint, headache limited to the temporal region ${ }^{4}$ and otalgia or tinnitus, or both in the absence of aural disease. The correlation between pathological changes in the joints or muscles and selfreported severity of pain is poor. ${ }^{5}$

Myalgia presents as a pain, dull aching in character, due to injury or repetitive strain of muscles. Myalgias are usually acute, though, with continued muscle strain, can occur for longer periods of time. Myofascial pain (MFP) also presents as a continuous dull aching pain that varies in intensity and duration. The MFP can be elicited, by palpating the muscle, that is local and may also refer to other sites, as mapped out by Simons et al. ${ }^{6}$ MFP is chronic in nature, seen in muscles which are under continuous strain. In MFP a taut band can often be localized to muscles known as trigger points. These trigger points, when stimulated, can result in a local twitch response often associated with decreased muscle length. ${ }^{7}$ The trigger points can be palpated to elicit patients pain response, thus confirming diagnosis. A vapocoolant spray or local anesthetic injection, can be used to block the source of the pain (i.e, masseter muscle), this may also be helpful in reaching definitive diagnosis.

A localized transient inflammation involving the muscle and fascial tissues is termed Myositis. ${ }^{8}$ The pain tends to increase with mandibular movements and localized tenderness, usually following injury or infection.

The present study was conducted to evaluate and compare the efficacy of topically applied and systemically administered NSAIDs in the management of TMD origin pain. The study divided the participants into 2 categories: 
Group A: oral diclofenac $50 \mathrm{mg}$, and Thiocolchicoside 4mg twice daily for 15 days.

Group B: 200mg diclofenac transdermal patch applied over masseter muscle [Fig. 1] 12 hrly and Thiocolchicoside 4mg twice daily for 15 days.

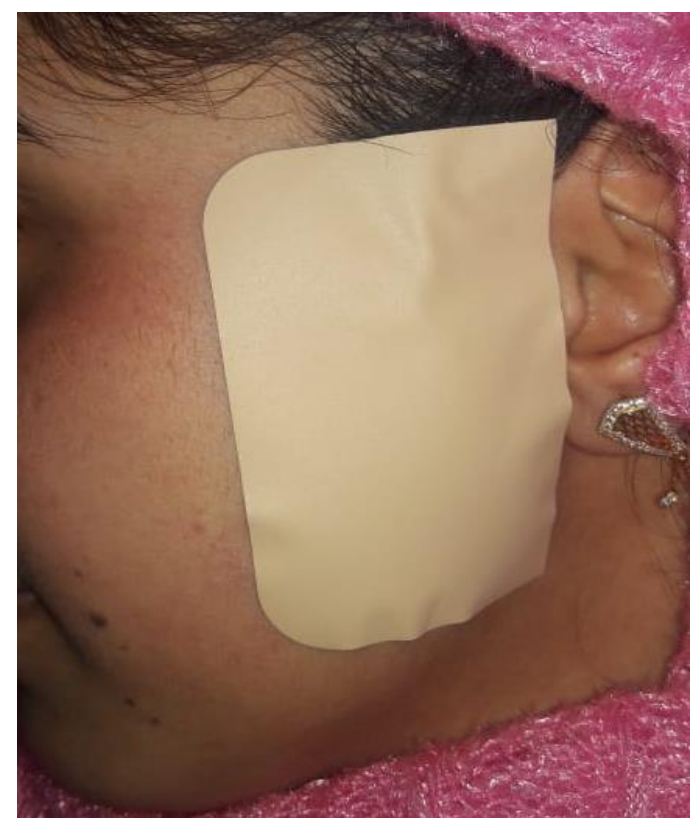

Fig. 1

\section{Aim}

To evaluate the efficacy of oral versus transdermal application of NSAID'S in treating muscular pain resulting from TMD.

\section{Objectives}

1. To compare the efficacy of topical verses systemic NSAID in treatment of pain associated with TMJ disorders.

2. To evaluate whether application of transdermal patch is better in terms of complications/side effects and compliance as compared to the oral NSAIDS.

\section{Materials and Methods}

A single blinded cross-sectional study was conducted in the Department of Oral Medicine and Radiology and a total of 40 clinically diagnosed cases of TMDs of muscular origin were selected. All the relevant information regarding demographic data, detailed history and clinical findings were recorded in a structured proforma after taking a written informed consent for the clinical drug trial. A detailed examination of masticatory muscles and accessory muscles associated with mandibular movements are an integral part of identifying the source of pain.

\section{Inclusion Criteria}

1. Patients with signs and symptoms of muscular pain of TMD origin according to the Research Diagnostic Criteria for Temporomandibular Disorder (RDCTMD). ${ }^{9}$
2. Psychologically healthy subjects who were fit to answer the questionnaire.

\section{Exclusion Criteria}

1. Patients with diseases involving TMJ like osteoarthritis, rheumatoid arthritis, gout etc

2. Patients with systemic diseases like hypertension, diabetes, asthma, epilepsy.

3. Patients with connective tissue disorders like SLE, scleroderma.

4. Patients with past history of TMD treatment.

5. Patients with history of trauma to TMJ and maxillofacial region or malignancy of maxillofacial region.

6. Patients allergic to diclofenac or Thiocolchicoside.

7. Patients who have gastritis, peptic or duodenal ulcers.

Once the diagnosis of myalgia due to TMD was made, the patients were randomly allocated in one of the treatment groups. The study divided the participants into 2 categories:

Group A: Oral diclofenac 50mg, and Thiocolchicoside $4 \mathrm{mg} 12$ hourly for 15 days.

Group B: 200mg of transdermal patch of diclofenac to be applied over the masseter muscle 12 hourly and Thiocolchicoside $4 \mathrm{mg}$ twice daily as in group A for the period of 15 days.

Before the commencement of the study a written consent was taken from each participant. Patients were instructed to note down their NRS [numeric rating scale] score twice a day in a diary daily during first week which constituted the subjective assessment.

Patients were recalled on $3^{\text {rd }}, 7^{\text {th }}, 15^{\text {th }}$ day and 21 th day. Drugs were discontinued after 15th day; subjective assessment (pain on NRS) was done on follow up visits on $7^{\text {th }}, 15^{\text {th }}$ and 21 th day.

\section{Statistical Methods}

Statistical software's SPSS (Version 20.0) and Microsoft Excel were used to carry out the statistical analysis of data. Data were analyzed by means of descriptive statistics, viz., percentages and means. Graphically, the data were presented by line graphs and doughnut diagram. $\mathrm{P}<0.05$ was considered statistically significant.

\section{Results}

In our study, total of 40 patients were included with the youngest being 21-year-old and the oldest being 61 years of age with mean age of 33.5 years. These findings were consistent with Okeson, 2003 which also states that commonly affected age group is 2 nd and 3rd decade of life. ${ }^{10}$ Total number of females in our study were $27[66 \%]$ and males 13 [33\% \} with a ratio of 2.07:1 (Graph 1), which correlates with study done by Riden in $1986 .{ }^{11}$ 


\section{Graph 1}

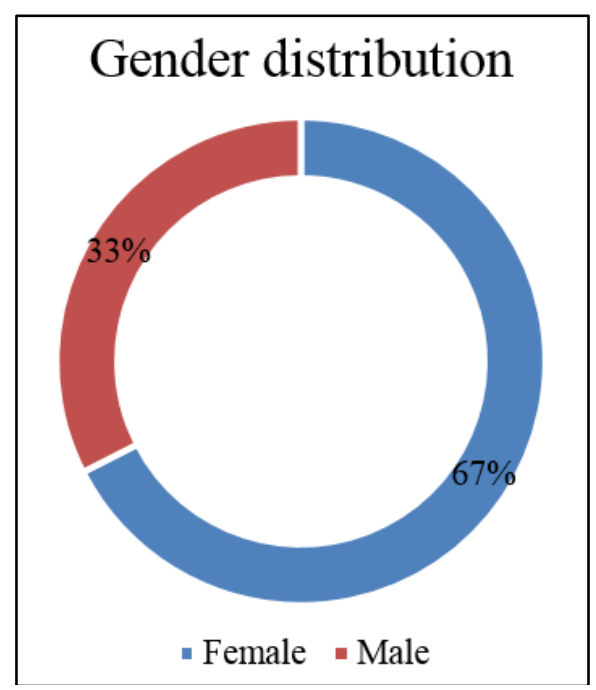

Group A consisted of 20 patients among which 14 were females and 6 were males in age range of 21 to 61 years. . Mean verbal rating score of pain was $8,5,4,1$ on $3^{\text {rd }} 7^{\text {th }}$, $15^{\text {th }}$, and 21 ist day respectively.

\section{Graph 2}

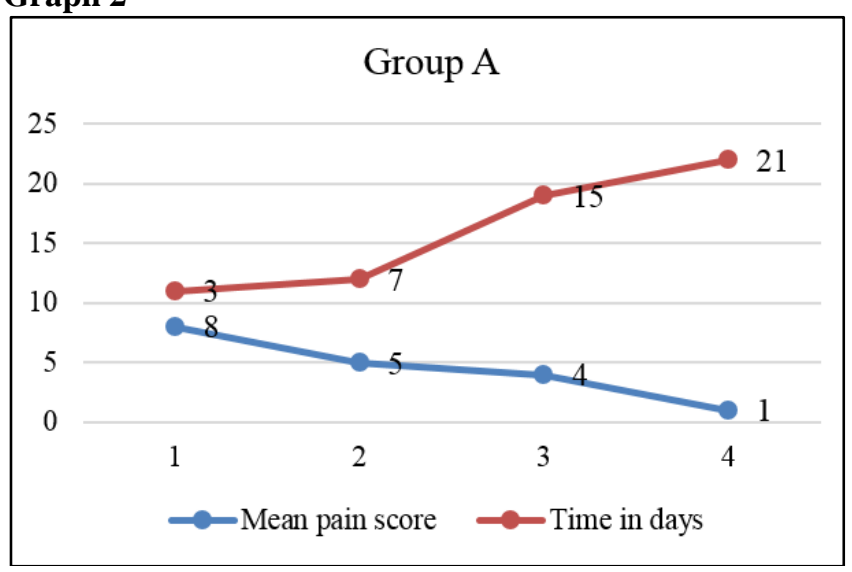

Group B also consisted of 20 patients having 7 male and 13 females age range of 23 to 53 years. Mean verbal rating score of pain was $7,5,3,1$ on $3^{\text {rd }}, 7^{\text {th }}, 15^{\text {th }}$, and 21 ist day respectively.

Graph 2 and Graph 3 depicting mean pain according to Numeric rating score in Group A and Group B. There was no significant difference in trends of pain intensity in oral NSAIDS group [Group A] and Transdermal patch group [Group B].

Treatment with topical NSAIDs has been shown to provide clinically effective analgesia at the site of application while minimizing systemic absorption. ${ }^{12,13}$ The benefits associated with topical NSAIDs include: (1) avoidance of first-pass metabolism and other variables associated with the absorption of drugs through the gastrointestinal tract; (2) reduced systemic side effects; (3) ease of dose termination in the event of adverse events; (4) sustained and controlled drug delivery over an extended period of time; (5) direct access to target site; (6) convenient administration; (7) improved patient acceptance and adherence; and (8) a viable solution for treatment when oral dosing is not possible. ${ }^{14}$

\section{Discussion}

The orofacial pain classification as outlined by Okeson ${ }^{15,16}$ is divided into physical (Axis 1) and psychological (Axis 2) conditions. Physical conditions comprise temporomandibular disorders (TMD), which include disorders of the temporomandibular joint (TMJ) and disorders of the musculoskeletal structures (eg, masticatory muscles and cervical spine); neuropathic pains, which include episodic (eg, trigeminal neuralgia [TN]) and continuous (eg, peripheral/centralized mediated) pains and neurovascular disorders (eg, migraine). Psychological conditions include mood and anxiety disorders.

It is well-recognized in the literature that temporomandibular disorders involve the biopsychosocial sphere as well, with chronic pain and functional limitation. Anxiety, depression and somatization disorders have been associated to TMD symptoms, and early findings suggesting a different psychosocial impairment between the subgroups of TMD patients ${ }^{17,18}$ have been dismounted by recent suggestions that pain, regardless of its muscular or articular origin, is the fundamental factor that relates TMD to the presence of depressive symptoms. ${ }^{19}$ The relation between duration of pain and severity of psychosocial impairment has also been investigated and, even in the absence of definite conclusions, there is preliminary support to the hypothesis that depressive disorders seem to be mostly associated with chronic TMD and anxiety disorders with the acute phase of symptom onset. ${ }^{20,21}$

The management starts with a detailed history and examination of patient and quantification of pain on a graded scale (Visual Analog Score" VAS"). The treatment modalities include surgical and non-surgical methods. In this study non surgical method was used. NSAIDs have been the mainstay in pain management. The efficacy of muscle relaxants in alleviating myalgia is well documented. Skeletal muscle relaxants are classified into two: antispastic and antispasmodic medications. The site of action of antispastic agents is the spinal cord or they may act directly on the skeletal muscles there by improving muscle hypertonicity and involuntary spasms whereas antispasmodics alter CNS conduction there by decreasing muscle spasms. Thiocholchicoside an antispasmodic agent is indicated for symptomatic treatment of muscle spasms and pain associated with musculoskeletal conditions like myalgia. Patient education about restricting mandiular movements and stress, home exercises, habit awareness and modification are important for a satisfactory outcome of treatment. The treatment includes taking the psychological aspect of the TMD also into consideration. 


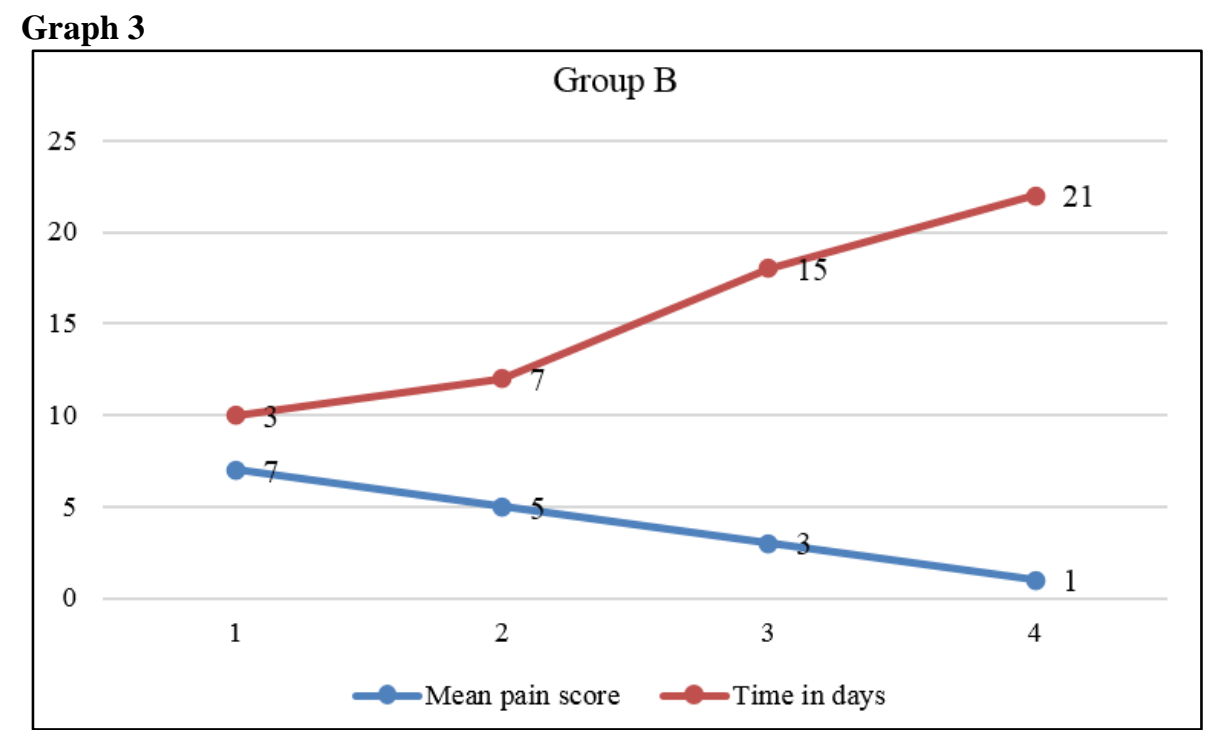

Thiocolchicoside (THC) is used clinically for its muscle relaxant, anti-inflammatory, and analgesic properties, it is also used topically for the treatment of muscular spasms and for rheumatologic, orthopedic, and traumatologic disorders. Thiocolchicoside binds to GABA-A and strychnine sensitive glycine receptors. Thiocolchicoside acting as a GABA-A receptor antagonist, its myorelaxant effects could be exerted at the supra-spinal level, via complex regulatory mechanisms, although a glycinergic mechanism of action cannot be excluded. The characteristics of the interaction of Thiocolchicoside with GABA-A receptors are qualitatively and quantitatively shared by its main circulating metabolite, the glucuronic dated derivative. Thiocolchicoside is rapidly absorbed after oral administration, and metabolized into 3 main metabolites. The two main circulating forms were the Thiocolchicoside aglycon and the glucuronic dated derivative of Thiocolchicoside, which is active. Thiocolchicoside is well tolerated oral administration for periods of up to 6 months. ${ }^{22-24}$

Transdermal diclofenac patch contains $1.3 \%$ diclofenac epolamine $(200 \mathrm{mg})$, adhesive material applied to a nonwoven polyester felt backing and covered with a polypropylene film release liner. The release liner is removed and the patch is then applied topically to the skin. The transdermal patch applied to intact skin provides local analgesia by releasing diclofenac epolamine from the patch into the skin. Diclofenac is an aryl acetic acid group nonsteroidal anti-inflammatory drug (NSAID). In pharmacologic studies, diclofenac has shown antiinflammatory, analgesic, and antipyretic activity. As with other NSAIDs, its mode of action is not known; its ability to inhibit prostaglandin synthesis, however, may be involved in its anti-inflammatory activity, as well as contribute to its efficacy in relieving pain associated with inflammation.

\section{Conclusion}

No significant difference was found in trends of pain intensity in patients treated by oral NSAIDS and patients who were treated with Transdermal patch group. However treatment with topical NSAIDs has been shown to provide clinically effective analgesia at the site of application while minimizing systemic absorption. The benefits associated with topical NSAIDs include: (1) avoidance of first-pass metabolism and other variables associated with the absorption of drugs through the gastrointestinal tract; (2) reduced systemic side effects; (3) ease of dose termination in the event of adverse events; (4) sustained and controlled drug delivery over an extended period of time; (5) direct access to target site; (6) convenient administration; (7) improved patient acceptance and adherence; and (8) a viable solution for treatment when oral dosing is not possible. ${ }^{14}$

\section{Conflict of Interest: None.}

\section{References}

1. Dimitroulis G. Temporomandibular disorders in general practice [Internet]. [Cited 2016 July 21]. Available from: http://epworthgp.org.au/temporomandibular-disorders-ingeneral-practice/.

2. Greene CS. Managing the care of patients with temporomandibular disorders: a new guideline for care. $J \mathrm{Am}$ Dent Assoc 2010;141:1086-8.

3. Arora P, Goswami R, Jain RS. The enigma of myofascial pain dysfunction syndrome. Int J Adv Sci Res 2015;1:1-4.

4. Schiffman E, Ohrbach R, List T, Anderson G, Jensen R, John MT, et al. Diagnostic criteria for headache attributed to temporomandibular disorders. Cephalalgia 2012;32:683-92.

5. Cairns B, List T, Michelotti A, Ohrbach R, Svensson P. JORCORE recommendations on rehabilitation of temporomandibular disorders. J Oral Rehabil 2010;37:481-9.

6. Simons DG, Travel JG, Simons LS. Myofascial Pain and Dysfunction: The Trigger Point Manual. Upper Half of Body. 2nd ed. Atlanta, GA: Lippincott Williams \& Wilkins; 1998;1.

7. Sanitá PV, de Alencar Júnior FGP. Myofascial pain syndrome as a contributing factor in patients with chronic headaches. $J$ Musculoskelet Pain 2009;17(1):15-25.

8. de Leeuw R. Temporomandibular Disorders. In: de Leeuw R, editor. Orofacial Pain Guidelines for Assesment, Diagnosis and Management. The American Academy of Orofacial Pain. 4th ed. Hanover Park, IL: Quintessence Publishing Co, Inc; 2008:158-76. 
9. Schiffman E. The Revised Research Diagnostic Criteria for Temporomandibular Disorders: Methods used to Establish and Validate Revised Axis I Diagnostic Algorithms. J Orofac Pain 2010;24(1):63-78.

10. Okeson JP. Management of Temporomandibular Disorders and Occlusion. 5th ed. St. Louis, MO: CV Mosby;2003. p. 8.

11. Riden DK. A clinical approach to pain: 2. Extra oral structures. Dent Update 1986;13:439-46:448-9.

12. McCarberg BH, Argoff CE. Topical diclofenac epolamine patch $1.3 \%$ for treatment of acute pain caused by soft tissue injury. Int J Clin Pract. 2010;64(11):1546-53.

13. Bookman AA, Williams KS, Shainhouse JZ. Effect of a topical diclofenac solution for relieving symptoms of primary osteoarthritis of the knee: a randomized controlled trial. CMAJ 2004;171(4):333-8.

14. Stanos SP. Topical agents for the management of musculoskeletal pain. J Pain Symptom Manage 2007;33(3):342-55.

15. Okeson JP. Bell's Orofacial Pains. The Clinical Management of Orofacial Pain. 6th ed. Carol Stream, IL: Quintessence Publishing Co, Inc; 2005.

16. Okeson JP. The Classification of Orofacial Pains. Oral Maxillofac Surg Clin North Am 2008;20(2):133-44.

17. Rugh JD, Woods BJ, Dahlstrom L. Temporomandibular disorders: assessment of psychological factors. Adv Dent Res 1993; 7:127-36.

18. Kight M, Gatchel RJ, Wesley L. Temporomandibular disorders: evidence for significant overlap with psychopathology. Health Psychol 1999;18:177-82.

19. Manfredini D, Bandettini di Poggio A, Romagnoli M, Dell' OssoL, Bosco M. Mood spectrum in patients with different painful temporomandibular disorders. Cranio 2004;22:234-40.
20. Gatchel RJ, Garofalo JP, Ellis E, Holt H. Major psychological disorders in acute and chronic TMD: an initial examination. $J$ Am Dent Assoc 1996;127:1365-74.

21. Epker J, Gatchel RJ. Coping profile differences in the biopsychosocial functioning of patients with temporomandibular disorder. Psychosom Med 2000;62:69-75.

22. Maryadele J. O'Neil, Ann Smith, Patricia E. Heckelman, 2001; "The Merck Index"; Thirteenth edition; Merck \& Co.,Inc.,Whitehouse Station,NJ.,USA;1662,1663

23. http://www.chembase.com/cbid_72067.htm11)http://www.agri culturesource.com/p-gloriosa-superba-seeds-and-rhizomesgloriosa-seeds-for-colchicine-and-thiocolchicoside996990.html

24. http://www.google.co.in/search?q=description+of+thiocolchic oside\&hl=en

How to cite this article: Hussain A, Ahmad R, Nazir N. Efficacy of transdermal diclofenac patches in the treatment of pain associated with various temporomandibular joint disorders: A comparative study. J Oral Med, Oral Surg, Oral Pathol, Oral Radiol 2019;5(2):37-41. 